Vol. XX (XXXX)

No. X

\title{
A GENERAL CONSTRUCTION OF POISSON BRACKETS ON EXACT MULTISYMPLECTIC MANIFOLDS
}

\author{
Michael Forger \\ Departamento de Matemática Aplicada, \\ Instituto de Matemática e Estatística, \\ Universidade de São Paulo, \\ Caixa Postal 66281, \\ BR-05311-970 São Paulo, S.P., Brazil \\ forger@ime.usp.br \\ Cornelius Paufler Hartmann Römer \\ Physikalisches Institut \\ Fakultät für Mathematik und Physik \\ Albert-Ludwigs-Universität Freiburg im Breisgau \\ Hermann-Herder-Straße 3 \\ D-79104 Freiburg i.Br., Germany \\ cornelius.paufler@physik.uni-freiburg.de_hartmann.roemer@physik.uni-freiburg.de \\ (Received 2002)

\begin{abstract}
In this note the long standing problem of the definition of a Poisson bracket in the framework of a multisymplectic formulation of classical field theory is solved. The new bracket operation can be applied to forms of arbitary degree. Relevant examples are discussed and important properties are stated with proofs sketched.
\end{abstract}

Keywords: Multisymplectic geometry, classical field theory, Poisson bracket

\section{Introduction}

Multisymplectic geometry provides a mathematical framework to describe classical field theory geometrically. Within this formulation it is not necessary to break manifest Lorentz covariance nor is there a need to use concepts from infinite dimensional geometry. The formalism dates back to the early work by De Donder, Dedecker, and Weyl. By now the exploration of its geometrical aspects has reached an elaborated stage, and a number of excellent reviews of this are available to the reader ([1]). In this article we propose an algebraic structure that mimics closely the Poisson algebra of classical mechanics.

Consider a classical field $\varphi^{i}(x), i=1, \ldots, N$ with $N$ internal degrees of freedom over $n$-dimensional space-time $\mathcal{M}$. Let $L\left(x^{\mu}, \varphi^{i}, \partial_{\mu} \varphi^{i}\right)$ denote the Lagrange density. The 
corresponding equations of motion are $\left(\frac{d}{d x^{\mu}}\right.$ denotes the total derivative w.r.t. $\left.x^{\mu}\right)$

$$
\frac{d}{d x^{\mu}}\left(\frac{\partial L}{\partial \partial_{\mu} \varphi^{i}}\right)-\frac{\partial L}{\partial \varphi^{i}}=0
$$

Introducing new field variables - the multimomenta - by

$$
\pi_{i}^{\mu}=\frac{\partial L}{\partial \partial_{\mu} \varphi^{i}}
$$

and the De Donder-Weyl Hamiltonian by

$$
H\left(x^{\mu}, \varphi^{i}, \pi_{i}^{\mu}\right)=\pi_{i}^{\mu} \partial_{\mu} \varphi^{i}-L
$$

one can easily verify that the equations of motion (1) are equivalent to the De DonderWeyl equations

$$
\frac{\partial H}{\partial \pi_{i}^{\mu}}=\frac{d}{d x^{\mu}} \varphi^{i} \quad, \quad \frac{\partial H}{\partial \varphi^{i}}=-\frac{d}{d x^{\mu}} \pi_{i}^{\mu},
$$

provided certain regularity conditions hold for $L$.

Note the remarkable similarity with the Hamiltonian equations of motion of mechanics. The difference one encounters lies in the fact that there are $n$ multimomenta associated to each degree of freedom $\varphi^{i}$. Of course, for $n=1$ one recovers the case of (time-dependent) Hamiltonian mechanics.

Furthermore, as the fields are sections of some possibly non-trivial fibre bundle $\mathcal{E} \rightarrow \mathcal{M}$, the notion of derivatives $\partial_{\mu} \varphi^{i}$ of the fields does not exist in a natural way. Rather, one has to use the theory of first jet bundles ( $[7]$ ) and - later on - their affine duals $J^{\star} \mathcal{E}$. For the sake of brevity we list the relevant objects occurring in multisymplectic geometry in a table, thereby comparing the individual items to their counterparts in the symplectic formulation of time-dependent mechanics.

\begin{tabular}{|r|l|}
\hline time-dependent mechanics & field theory \\
\hline extended configuration space & configuration bundle \\
$\mathcal{E}=\mathbb{R} \times \mathcal{Q}$ & $\mathcal{E} \rightarrow \mathcal{M}^{n}$ \\
\hline $\mathbb{R} \times T \mathcal{Q}$ & $J^{1} \mathcal{E}$ \\
\hline extended tangent space & first jet bundle \\
\hline $\mathcal{P}=\left(\mathbb{R} \times T^{*} \mathcal{Q}\right) \times \mathbb{R}$ & $P=J^{\star} \mathcal{E}$ \\
$\left(t, q^{i}, p_{i}, E\right)$ & $\left(x^{\mu}, q^{i}, p_{i}^{\mu}, p\right)$ \\
\hline canonical 1-form & multicanonical form \\
$\theta=p_{i} d q^{i}+E d t$ & $\theta=p_{i}^{\mu} d q^{i} \wedge d^{n} x_{\mu}+p d^{n} x$ \\
\hline symplectic form & multisymplectic form \\
$\omega=-d \theta=d q^{i} \wedge d p_{i}-d E \wedge d t$ & $\omega=-d \theta=d q^{i} \wedge d p_{i}^{\mu} \wedge d^{n} x_{\mu}-d p \wedge d^{n} x$ \\
\hline
\end{tabular}

The multicanonical form $\theta$ can be defined in an intrinsic way. We shall not elaborate on this but instead mention that there is a vector field $\Sigma$ on $\mathcal{P}$, the scaling or Euler vector field of the vector bundle $\mathcal{P} \rightarrow \mathcal{E}$,

$$
\Sigma=p_{i}^{\mu} \frac{\partial}{\partial p_{i}^{\mu}}+p \frac{\partial}{\partial p},
$$


that satisfies

$$
i(\Sigma) \omega=-\theta .
$$

$\mathcal{P}$, together with $\omega$ and $\theta$, is an example of an exact multisymplectic manifold. Note in particular that $\omega$ is non-degenerate on vector fields.

There is a natural projection from the extended multiphase space $\mathcal{P}$ to the ordinary multiphase space $\tilde{\mathcal{P}}$ given by (we just write down the coordinate expression)

$$
\mathcal{P} \rightarrow \tilde{\mathcal{P}}, \quad\left(x^{\mu}, q^{i}, p_{i}^{\mu}, p\right) \mapsto\left(x^{\mu}, q^{i}, p_{i}^{\mu}\right) .
$$

The dimension of $\tilde{\mathcal{P}}$ is one less than that of $\mathcal{P}$. The ordinary multiphase space has been used by Kanatchikov (河), Echeverría-Enríquez et al. ([2]) in their work. It shows the unpleasant feature of not carrying a canonical multisymplectic structure. However, with the help of connections in $\mathcal{E}$ and $T \mathcal{M}$, the analogues $\theta^{V}$ and $\omega^{V}$ of the above items can be defined and one has

$$
\omega^{V}=-d^{V} \theta^{V},
$$

where $d^{V}$ is an exterior derivative along the fibres of $\tilde{\mathcal{P}} \rightarrow \mathcal{M}$ acting on forms on $\tilde{\mathcal{P}}$.

\section{Poisson forms}

It is natural to study solutions of the equation

$$
i\left(X_{f}\right) \omega=d f .
$$

As the multisymplectic form $\omega$ is an $(n+1)$-form, $f$ can be an $r$-form, $r=0,1, \ldots,(n-1)$. Correspondingly, $X_{f}$ has to be an $(n-r)$-vector field, $X_{f} \in \Lambda^{n-r} \mathfrak{X}(\mathcal{P})$. Note that $\omega$ is degenerate on multi-vector fields of tensor degree higher than 1 (otherwise it would have to be the volume form on $\mathcal{P}$ ). Consequently, $X_{f}$ is not uniquely determined by $f$, nor is $f$ fixed by $X_{f}$. Moreover, it is known that there are conditions on $f$ which have to be met for an $X_{f}$ to exist. These restrictions concern the dependence of the coefficients of $F$ on the multimomenta $p_{i}^{\mu}$. If the pair $\left(X_{f}, f\right)$ forms a solution of (9) then $f$ is called Hamiltonian form and $X_{f}$ Hamiltonian multi-vector field. With the definition of the Lie derivative along an $r$-vector field $Y$,

$$
L_{Y}=d i(Y)-(-1)^{r} i(Y) d
$$

one can formulate necessary and sufficient conditions for an multi-vector field $X$ to be a Hamiltonian multi-vector field associated to some Hamiltonian form. More precisely, if $X$ denotes such a Hamiltonian multi-vector field, then

$$
L_{X} \omega=0,
$$

and if

$$
L_{X} \theta=0
$$


then $X$ is a Hamiltonian $\mathrm{r}$-vector field associated to

$$
f=J(X)=(-1)^{r} i(X) \theta .
$$

Multi-vector fields satisfying (12) are called exact Hamiltonian multi-vector fields. Typical examples of Hamiltonian forms will be discussed below.

As already mentioned the Hamiltonian multi-vector field $X_{f}$ is not fixed by $f$. However, as we shall see in a moment, the definition of a bracket operation between Hamiltonian forms will involve contractions of Hamiltonian multi-vector fields with Hamiltonian forms. This results in an undesirable dependence on the choice of the Hamiltonian multivector field. We therefore restrict the Hamiltonian forms further.

Definition 1. A Poisson form $f$ on $\mathcal{P}$ is a Hamiltonian form that in addition satisfies

$$
i(Y) \omega=0 \quad \Rightarrow \quad i(Y) f=0
$$

for all multi-vector fields $Y$ on $\mathcal{P}$. Equivalently, for a Poisson form $f$ there exists a multi-vector field $Z$ on $\mathcal{P}$ with

$$
i(Z) \omega=f .
$$

Remark. For exact Hamiltonian $r$-vector fields $X_{f}$, one has

$$
J\left(X_{f}\right)=i\left(X_{f} \wedge \Sigma\right) \omega .
$$

To show that the notion of a Poisson form is non-empty we give a list of examples that have been discussed in the literature.

Functions on $\mathcal{P}$ are Poisson. If in addition a function $f$ is of the form

$$
f\left(x^{\mu}, q^{i}, p_{i}^{\mu}, p\right)=-H\left(x^{\mu}, q^{i}, p_{i}^{\mu}\right)-p,
$$

then it admits a Hamiltonian $n$-vector field $X$ that is locally decomposable,

$$
X=Z_{1} \wedge \cdots \wedge Z_{n}, \quad Z_{\mu} \in \mathfrak{X}(\mathcal{P}) .
$$

The solutions of the De Donder-Weyl equations with $H$ as above define a tangent space at every point of $\mathcal{P}$ that is spanned by the collection of the $Z_{\mu}$. The converse, i.e. the integration of the $Z_{\mu}$ to a solution of the De Donder-Weyl equations can be performed if additional conditions are fulfilled (for details, see [6]).

A form $\tilde{f}$ on the ordinary multiphase space $\tilde{\mathcal{P}}$ is called horizontal if it vanishes on vector fields that are vertical w.r.t. the projection onto $\mathcal{M}$. Kanatchikov has studied such horizontal forms on $\tilde{\mathcal{P}}$ that in addition satisfy

$$
i\left(\tilde{X}_{\tilde{f}}\right) \omega^{V}=d^{V} \tilde{f} .
$$

In the sequel we will refer to such forms as Hamiltonian forms à la Kanatchikov. One can show that they are in $1-1$ correspondence with horizontal Hamiltonian forms $f$ on $\mathcal{P}$, where $f$ is the pullback of $\tilde{f}$. Obviously, horizontal Hamiltonian forms on $\mathcal{P}$ are Poisson. 
Let $\xi_{\mathcal{E}}$ be a vector field on the configuration bundle $\mathcal{E}$ which is projectable onto $\mathcal{M}$. There is a canonical lift of $\xi_{\mathcal{E}}$ to $\mathcal{P}$. The resulting vector field $\xi_{\mathcal{P}}$ is the generator of a special canonical transformation on $\mathcal{P}$. One has

$$
L_{\xi_{\mathcal{P}}} \theta=0 .
$$

Consequently,

$$
J\left(\xi_{\mathcal{P}}\right)=i\left(\xi_{\mathcal{P}}\right) \theta
$$

is Poisson. Moreover, let $X_{1}, X_{2}$ be commuting exact Hamiltonian vector fields. Then one can show

$$
L_{X_{1} \wedge X_{2}} \theta=0,
$$

and one obtains examples $J\left(X_{1} \wedge X_{2}\right)$ of Poisson forms of intermediate tensor degree. These forms can be viewed as the counterparts to higher dimensional orbits of transformations.

There is a canonical projection from Hamiltonian $(n-r)$-forms onto Poisson $(n-r)$ forms with exact Hamiltonian $r$-vector fields, given by

$$
f \mapsto(-1)^{r-1} i\left(X_{f}\right) \theta, \quad X_{f} \mapsto X_{f}+\left[\Sigma, X_{f}\right] .
$$

Obviously, this map does have a kernel. However, it has not been clarified yet whether there exist Hamiltonian multi-vector fields that are not Poisson.

\section{Poisson brackets}

Before we turn to the definition of a bracket operation between Poisson forms let us briefly recall the canonical extension of the Lie-bracket of vector fields to the algebra of multi-vector fields. This composition is known under the name Schouten bracket. The

defining formulæ are (let $X, Y, Z$ be multi-vector fields of respective tensor degree $r, s, t$ )

$$
\begin{aligned}
{[X, Y \wedge Z] } & =[X, Y] \wedge Z+(-1)^{(r-1) s} Y \wedge[X, Z], \\
{[Y, X] } & =-(-1)^{(r-1)(s-1)}[X, Y] .
\end{aligned}
$$

Moreover, one requires that it coincide with the standard Lie bracket on vector fields. This fixes the bracket. An important consequence of these properties is a graded Jacobi identity

$$
(-1)^{(r-1)(t-1)}[X,[Y, Z]]+\text { cyclic perm. }=0 .
$$

In addition, one can derive the following formula relating Lie derivative and Schouten bracket:

$$
L_{[X, Y]}=(-1)^{(r-1)(s-1)} L_{X} L_{Y}-L_{Y} L_{X} .
$$


Now lets turn to the definition of a bracket operation for Poisson forms. Let $f, g$, $h$ be Poisson forms with associated Hamiltonian multi-vector fields $X_{f}, X_{g}$, and $X_{h}$ of respective tensor degree $r, s, t$.

An obvious ansatz for the Poisson bracket is given by

$$
\{f, g\}^{\prime}=(-1)^{r} i\left(X_{f}\right) i\left(X_{g}\right) \omega .
$$

This formula has been suggested by many authors. It shows the desired properties

$$
\begin{aligned}
\{f, g\}^{\prime} & =-(-1)^{(r-1)(s-1)}\{g, f\}^{\prime} \\
X_{\{f, g\}^{\prime}} & =\left[X_{g}, X_{f}\right] .
\end{aligned}
$$

However, it does not satisfy a graded Jacobi identity that meets with (25):

$$
(-1)^{(r-1)(t-1)}\left\{f,\{g, h\}^{\prime}\right\}^{\prime}+\text { cycl. perm. }=(-1)^{(t-1)(r-1)+s} d\left(i\left(X_{f}\right) i\left(X_{g}\right) i\left(X_{h}\right) \omega\right) .
$$

Moreover, hideous additional terms occur in the composition of two generators of transformations (let $X, Y$ be exact Hamiltonian multi-vector fields of degree $r$ and $s$ ),

$$
\{J(X), J(Y)\}^{\prime}=J([Y, X])-(-1)^{(s-1) r} d(i(Y) i(X) \theta .
$$

If $d$ has trivial cohomology one can use (30) as a starting point for the construction of an $l(\infty)$-structure.

We propose a modification of (27) which preserves (28) and (29) but cures the anomalous terms in (30) and (31).

Definition 2. Let $f$ and $g$ be Poisson forms with respective Hamiltonian multivector fields $X_{f}$ and $X_{g}$. Then their bracket $\{f, g\}$ is given by

$$
\{f, g\}=-L_{X_{f}} g+(-1)^{(r-1)(s-1)} L_{X_{g}} f-(-1)^{(r-1) s} L_{X_{f} \wedge X_{g}} \theta,
$$

Where $X_{f}$ and $X_{g}$ are multi-vector fields of degree $r$ and $s$, respectively. Equivalently,

$$
\begin{aligned}
\{f, g\}= & (-1)^{r} i\left(X_{g}\right) i\left(X_{f}\right) \omega \\
& +d\left((-1)^{(r-1)(s-1)} i\left(X_{g}\right) f-i\left(X_{f}\right) g-(-1)^{(r-1) s} i\left(X_{g}\right) i\left(X_{f}\right) \theta\right) .
\end{aligned}
$$

Proposition 1. Let $f, g, h$ be Poisson forms with respective Hamiltonian $r$-, $s$-, and $t$-vector fields $X_{f}, X_{g}$ and $X_{h}$. Then the following properties hold.

1. The bracket $\{f, g\}$ does not depend on the particular choice of $X_{f}$ and $X_{g}$.

2. $X_{\{f, g\}}=\left[X_{g}, X_{f}\right]$.

3. The bracket $\{f, g\}$ of two Poisson forms $f, g$ is again a Poisson form. 
4. The bracket is graded antisymmetric,

$$
\{f, g\}=-(-1)^{(r-1)(s-1)}\{g, f\} .
$$

5. It satisfies a graded Jacobi identity,

$$
(-1)^{(r-1)(t-1)}\{f,\{g, h\}\}+\text { cyclic perm. }=0 .
$$

6. For exact Hamiltonian multi-vector fields $X, Y$, one has

$$
\{J(X), J(Y)\}=J([Y, X]) .
$$

7. Let $\tilde{f}, \tilde{g}$ be Hamiltonian forms à la Kanatchikov, $\tilde{X}_{\tilde{f}}$ and $\tilde{X}_{\tilde{g}}$ be their multi-vector fields (of tensor degree $r$ and s) according to (19). Then $i\left(\tilde{X}_{\tilde{f}}\right) i\left(\tilde{X}_{\tilde{g}}\right) \omega^{V}$ is again Hamiltonian à la Kanatchikov and can be pulled back to $\mathcal{P}$, where it coincides with the bracket $\{f, g\}$ of the pulled back forms $f$ and $g$.

Proof. While the proofs of items 1,2,3,4,6 are obvious, the demonstration of the graded Jacobi identity is rather lengthy. It follows from the properties (25) and (26). A calculation in coordinates shows the last statement. The details of the proofs are contained in $[3]$.

Remark. The definition and the proposition (except the last statement) do not involve any properties other than

$$
\omega=-d \theta, \quad i(\Sigma) \omega=-\theta .
$$

Hence the construction can be carried over to arbitrary exact multisymplectic manifolds, in particular to the direct treatment of higher order Lagrangeans (顿).

The notion of a Poisson bracket is not completely justified as no product structure has been identified yet. One can show that a horizontal Poisson form of degree $r$ has to be polynomial in the multimomenta of degree $(n-r)$ or less. Hence, the product of a Poisson $r$-form and a Poisson $s$-form can be at most an $(r+s-n)$-form. There are two candidates for such a composition: Kanatchikov's $\bullet$-product

$$
f \bullet g=*^{-1}(* f \wedge * g),
$$

where $*$ is a Hodge operation on $\mathcal{M}$, acting on horizontal forms on $\tilde{\mathcal{P}}$, and - on the side of multi-vector fields - the map

$$
X, Y \mapsto X \wedge Y .
$$

One can show that both maps do not coincide. While the former does yield a Poisson form and satisfies a graded Leibniz rule, the latter cannot be carried over to Poisson forms. This can be seen from the identity

$$
L_{X_{f} \wedge X_{g}} \omega= \pm d\{f, g\} .
$$

The $\bullet$-product, however, cannot be extended to general Poisson forms without the use of a connection. Moreover, the product of two functions will always give zero which makes it impossible to view it as a generalisation of the product of functions in symplectic geometry. 


\section{Conclusions}

Multisymplectic geometry provides a covariant formalism for classical field theory. It uses elements from finite dimensional differential geometry throughout. There is a close resemblance to the formulation of time dependent Hamiltonian mechanics. However, there are a number of features that do not occur in mechanics.

In particular there is a correspondence between forms and multi-vector fields that replaces the interplay of functions and Hamiltonian vector fields of symplectic geometry. Forms of degree $n-1$, where $n$ is the dimension of space-time, come in very naturally when considering symmetry transformations. Remember that according to Noether's theorem there is a conserved charge for every symmetry, and this charge is obtained through integration over $(n-1)$-dimensional hypersurfaces of space-time. On the other hand, as solutions of the field equations are objects of dimension higher than 1 , we expect $n$-vector fields to replace the Hamiltonian vector fields of mechanics. This is indeed the case.

These considerations show that there is a need to have a (Poisson) bracket operation for forms at hand, and in view of later quantisation attempts this structure should obey a Jacobi identity. In this note we have proposed such a bracket for Poisson forms. We have shown that all relevant examples occurring in the geometrical formulation of classical field theories are indeed Poisson forms. Moreover, the proposed operation does satisfy a graded Jacobi identity that is closely related to the Jacobi identity of the Schouten bracket of multi-vector fields. We have shown that the bracket operation closes on Poisson forms and contains as a particular case the Gerstenhaber algebra introduced by Kanatchikov (河). Furthermore, with this structure at hand, one is now able to algebraically investigate symmetry transformations, including space-time translations.

The construction is independent of the particular structure of the exact multisymplectic manifold under consideration. Therefore, it is possible to apply the results to field theories beyond first order Lagrangeans, i.e. to gravity as an example. It would

be of interest to study the treatment of constraints in this context. These matters are currently under investigation.

\section{REFERENCES}

[1] J.F. Cariñena, M. Crampin \& L.A. Ibort: On the Multisymplectic Formalism for First Order Field Theories, Diff. Geom. Appl. 1 (1991) 345-374.

M.J. Gotay, J. Isenberg \& J.E. Marsden: Momentum Maps and Classical Relativistic Fields I: Covariant Field Theory, physics/9801019.

[2] A. Echeverría-Enríquez, M. C. Muñoz-Lecanda: Geometry of Multisymplectic Hamiltonian First-order Field Theories., J. Math. Phys. 41(11) (2000), 7402-7444, $\mathrm{dg}-\mathrm{ga} / 0004005$.

[3] M. Forger, C. Paufler \& H. Römer: The Poisson Bracket for Poisson Forms in Multisymplectic Field Theory, submitted for publication, math-ph/0202043.

[4] M. J. Gotay: A multisymplectic framework for classical field theory and the calculus of variations. I: Covariant Hamiltonian formalism. In M. Francaviglia und D. D. Holm (Hrsg.): Mechanics, analysis and geometry: 200 years after Lagrange, 203-235 (1991). 
[5] I.V. Kanatchikov: Canonical Structure of Classical Field Theory in the Polymomentum Phase Space, Rep. Math. Phys. 41 (1998) 49-90, hep-th/9709229.

[6] C. Paufler, H. RÖMer: Geometry of Hamiltonian n-vectors in Multisymplectic Field Theory. to appear in J. Geom. Phys., math-ph/0102008.

[7] D. J. Saunders: The Geometry of Jet Bundles. Lond. Math. Soc. Lect. Note Ser. 142, Cambr. Univ. Pr., Cambridge, 1989. 